LBL-37688

\title{
Thermal Equilibration in an Expanding Parton Plasma
}

\author{
H. Heiselberg \\ NORDITA, Blegdamsvej 17, DK-2100 Copenhagen Ø., Denmark \\ Xin-Nian Wang \\ Nuclear Science Division, Mailstop 70A-3307 \\ Lawrence Berkeley National Laboratory \\ University of California, Berkeley, CA 94720 USA
}

\begin{abstract}
Thermalization in an expanding parton plasma is studied within the framework of Boltzmann equation in the absence of any mean fields. In particular, we study the time-dependence of the relaxation time to the lowest order in finite temperature QCD and how such time-dependence affects the thermalization of an expanding parton plasma. Because of Debye screening and Landau damping at finite temperature, the relaxation time (or transport rates) is free of infrared divergencies in both longitudinal and transverse interactions. The resultant relaxation time decreases with time in an expanding plasma like $1 / \tau^{\beta}$, with $\beta<1$. We prove in this case that thermal equilibrium will eventually be established given a long life-time of the system. However, a fixed momentum cut-off in the calculation of the relaxation time gives rise to a much stronger time dependence which will slow down thermal equilibrium. It is also demonstrated that the "memory effect" of the initial condition affects the approach to thermal equilibrium and the final entropy production.
\end{abstract}

PACS numbers:12.38.Mh,25.75+r,12.38.Bx

${ }^{*}$ This work was supported by the Director, Office of Energy Research, Division of Nuclear Physics of the Office of High Energy and Nuclear Physics of the U.S. Department of Energy under Contract No. DE-AC03-76SF00098. 


\section{Introduction}

Perturbative-QCD-based models developed in the last few years predict that nucleusnucleus collisions at future collider energies are dominated by hard or semihard processes 11, 2, 3, 田. These processes happen during the very early stage of the collisions and they produce a rather large number of semihard partons which essentially form a hot and undersaturated parton gas «, 5]. However, this parton gas is initially far away from thermal and chemical equilibrium [6]. Secondary parton scatterings in the gas may eventually lead to local thermal and chemical equilibrium if the parton interactions are sufficiently strong.

Transport calculations based on a semiclassical parton cascade model [4] indicate that thermal equilibrium could be established within a rather short time of about $1 \mathrm{fm} / c$. However, the complexity of the Monte Carlo simulations makes it difficult to obtain a lucid understanding of the dependence of the thermalization time on the many parameters employed in the model. One such parameter is the cut-off of momentum transfer in binary parton scatterings. The cut-off was first introduced to regularize the infrared divergency of the cross section between two massless partons in high-energy $p p$ and $p \bar{p}$ collisions $[7]$. The value of the momentum cut-off is determined phenomenologically to reproduce the measured total cross sections of $p p$ and $p \bar{p}$ collisions. However, this cut-off is not necessary anymore in a high-temperature quark-gluon plasma, since the Debye screening and Landau damping provide natural regularizations of the infrared divergency. Since transport times depend sensitively on the screening masses which in turn depend on the temperature, the introduction of an artificial cut-off could give rise to a completely different behavior of the thermalization time and consequently the approach to thermal equilibrium.

The approach to thermal equilibrium in relativistic heavy ion collisions is dictated by the competition between expansion and parton interactions [8]. If the expansion is much rapid than the typical collision time among partons, e.g., shortly after partons are initially produced, the expansion is closer to free-streaming than hydrodynamic expansion. Only at times in the order of the collision time may the parton gas reach local thermal equilibrium and expand hydrodynamically. Furthermore, the time dependence of the collision time (or the relaxation time) will determine whether the system can eventually reach local thermal equilibrium because of the competition between expansion and parton interactions. If the collision time increases rapidly with time, the parton system may never thermalize, leading only to a free-streaming limit. The collision time, therefore, is a very important quantity which in turn depends sensitively on the infrared behavior of parton interactions.

In QCD, parton scattering cross sections exhibit a quadratic infrared singularity due to the exchange of a massless gluon. The infrared behavior can be improved by including corrections from hard thermal loops to the gluon propagators. Resummation of these thermal loops gives rise to an effective gluon propagator which screens long 
range interactions (Debye screening). Braaten and Pisarski [9] have developed this resummation technique systematically and used it to calculate the damping rate of a soft gluon $(p \sim g T)$ which is gauge invariant and complete to the leading order in the QCD coupling constant $g$ [10]. For a fast particle $(p \gtrsim T)$, the exchanged gluons probe the static limit of the magnetic interactions which by the transversality condition are not screened. One thus has to introduce a nonperturbative magnetic screening mass to regularize the logarithmic infrared singularity in the static limit [11, 12, 13]. As a result, the damping rate for an energetic particle to the leading order in $g$ is

$$
\Gamma \sim T\left[\alpha_{s} \ln \left(1 / \alpha_{s}\right)+\mathcal{O}\left(\alpha_{s}\right)\right]
$$

where $\alpha_{s}=g^{2} / 4 \pi$. However, as we will argue, damping rates do not determine how fast a system approach local thermal equilibrium. What really determine the thermalization processes are the transport rates which are free of the logarithmic divergency after the resummation of thermal loops [14, 15]. This is because thermalization is achieved to the leading order mainly through momentum changes in elastic scatterings. Thus, the effective cross section should be weighted by the momentum transfer and the dynamic screening due to the Landau damping of the gluons is sufficient to regularize the logarithmic singularity in the transverse interactions [16, 17, 18]. The resultant transport times for a system near thermal equilibrium behave like

$$
\frac{1}{\tau_{\mathrm{tr}}} \sim T \alpha_{s}^{2} \ln \left(1 / \alpha_{s}\right)
$$

to the leading order in $\alpha_{s}$.

For a system near local thermal equilibrium, the time dependence of the transport times is through the temperature according to Eq. (2). This dependence is in general slower than $1 / \tau$ and thus can lead to local thermal equilibrium according to our earlier argument based on the relaxation time approximation [8]. However, if one introduces an artificial cut-off for the momentum transfers of elastic parton scatterings as in the numerical simulation of a classical parton cascade [4], the time dependence will be much stronger. Consequently, as we will demonstrate in this paper, the system will approach local thermal equilibrium much slowly. We will also demonstrate that inclusion of the screening effects is the key to a slower time dependence of the relaxation time, therefore a faster approach to thermal equilibrium.

This paper is organized as follows. In Section II, we first re-examine the Boltzmann equation and the evaluation of the damping rate and the relaxation time to the lowest order, including only $2 \leftrightarrow 2$ processes. We will also discuss the time dependence of the relaxation time in different scenarios. In Section III, we will solve the Boltzmann equation in the relaxation time approximation and demonstrate how time dependence of the relaxation time will affect the approach to thermal equilibrium. We also show how initial conditions of a system affect the thermalization processes and the final total entropy production (or "memory effect") in Section IV. Finally in Section V 
we give a summary and an outlook, especially of the numerical simulations of parton thermalization, taking into account of the Debye screening and Landau damping effects without double counting.

\section{Time dependence of thermalization time}

In a system with two-components as, e.g., the quark and gluon plasma, the one that interacts the strongest will thermalize faster than the other. Subsequently there will be momentum and energy transfer between them. Since numerical simulations indicate that the initially produced partons are mostly gluon, we consider here a gluon gas only for simplicity. The Debye screening of color fields in the presence of semihard gluons [19] will also allow us to neglect the effect of mean fields. We furthermore assume that the spatial variation of the system is small on the scale of a collision length so that we can approximate the evolution of the system by the Boltzmann equation [20],

$$
\begin{aligned}
v_{1} \cdot \partial f_{1}\left(p_{1}\right) & =-\nu_{2} \int d p_{2} d p_{3} d p_{4} F_{1234}[f] \frac{1}{2}\left|M_{12 \rightarrow 34}\right|^{2}(2 \pi)^{4} \delta^{4}\left(P_{1}+P_{2}-P_{3}-P_{4}\right),(3) \\
F_{1234}[f] & =f_{1} f_{2}\left(1 \pm f_{3}\right)\left(1 \pm f_{4}\right)-f_{3} f_{4}\left(1 \pm f_{1}\right)\left(1 \pm f_{2}\right)
\end{aligned}
$$

where $P_{i}=\left(\left|p_{i}\right|, \mathbf{p}_{i}\right)$ are the four-momenta of massless partons and $d p_{i} \equiv d^{3} p /(2 \pi)^{3}$. To keep the formula general, \pm are used for bosons (gluons) and fermions (quarks and anti-quarks), respectively. The statistical factor $\nu_{2}$ is $2\left(N_{c}^{2}-1\right)=16$ for gluons and $12 N_{f}$ for $N_{f}$ flavors of quarks and anti-quarks with $N_{c}=3$ colors. The squared matrix element, $\left|M_{12 \rightarrow 34}\right|^{2} \equiv\left|\mathcal{M}_{12 \rightarrow 34}\right|^{2} /\left(16 E_{1} E_{2} E_{3} E_{4}\right)$ is summed over final states and averaged over initial states. For gluon-gluon scatterings,

$$
\left|\mathcal{M}_{12 \rightarrow 34}\right|^{2}=C_{g g} 4 g^{4}\left(3-\frac{s u}{t^{2}}-\frac{s t}{u^{2}}-\frac{t u}{s^{2}}\right)
$$

where $C_{g g}=N_{c}^{2} /\left(N_{c}^{2}-1\right)=9 / 8$ is the color factor of gluon-gluon scatterings, $s$, $t$ and $u$ are the Mandelstam variables. There is clearly a quadratic singularity for small energy $\omega$ and momentum $q$ transfers because of the long range interactions mediated by the massless gauge bosons. Because the final state has two identical particles, $s u / t^{2}$ should contribute equally as $s t / u^{2}$ in Eq. (5). We thus can approximate $\left|\mathcal{M}_{12 \rightarrow 34}\right|^{2} \approx 8 C_{g g} g^{4} s^{2} / t^{2}$ for small-angle gluon scatterings. Since the collisional integral is dominated by contributions from near the singularity, we can assume a small angle scattering approximation, i.e., $\omega, q \ll E_{1}, E_{2}$. Then energy-momentum conservation leads to

$$
\begin{aligned}
\mathbf{p}_{3} & =\mathbf{p}_{1}+\mathbf{q}, \quad \mathbf{p}_{4}=\mathbf{p}_{2}-\mathbf{q} \\
E_{3} & =E_{1}+\omega, \quad E_{4}=E_{2}-\omega \\
\omega & \approx \mathbf{v}_{\mathbf{1}} \cdot \mathbf{q} \approx \mathbf{v}_{\mathbf{2}} \cdot \mathbf{q} .
\end{aligned}
$$


The integration over $p_{3}$ and $p_{4}$ can be rewritten as

$$
(2 \pi)^{4} \int d p_{3} d p_{4} \delta^{4}\left(P_{1}+P_{2}-P_{3}-P_{4}\right)=\frac{1}{(2 \pi)^{2}} \int d^{3} q \int_{-q}^{q} d \omega \delta\left(\omega-\mathbf{v}_{\mathbf{1}} \cdot \mathbf{q}\right) \delta\left(\omega-\mathbf{v}_{\mathbf{2}} \cdot \mathbf{q}\right) .
$$

In a medium, one can use a resummation technique to include an infinite number of loop corrections to the gluon exchange. Using Dyson's equation, this amounts to an effective gluon propagator. One can use this effective propagator to obtain the effective matrix element squared for forward gluon scatterings (see Appendix A),

$$
\left|M_{g g}\right|^{2} \approx 2 C_{g g} g^{4}\left|\frac{1}{q^{2}+\pi_{L}(x)}-\frac{\left(1-x^{2}\right) \cos \phi}{q^{2}\left(1-x^{2}\right)+\pi_{T}(x)}\right|^{2},
$$

where $\cos \phi=\left(\mathbf{v}_{1} \times \hat{\mathbf{q}}\right) \cdot\left(\mathbf{v}_{1} \times \hat{\mathbf{q}}\right)$ and $x=\omega / q$. The scaled self-energies in the long wavelength limit are given by [21]

$$
\begin{aligned}
& \pi_{L}(x)=q_{D}^{2}\left[1-\frac{x}{2} \ln \left(\frac{1+x}{1-x}\right)+i \frac{\pi}{2} x\right] \\
& \pi_{T}(x)=q_{D}^{2}\left[\frac{x^{2}}{2}+\frac{x}{4}\left(1-x^{2}\right) \ln \left(\frac{1+x}{1-x}\right)-i \frac{\pi}{4} x\left(1-x^{2}\right)\right],
\end{aligned}
$$

where $q_{D}^{2}=g^{2}\left(N_{c}+N_{f} / 2\right) T^{2} / 3$ is the Debye screening mass in thermal QCD. The imaginary parts provide Landau damping to parton interactions in a thermal medium. We see that the longitudinal interactions are screened by thermal interactions. However, the transverse interactions still have a logarithmic singularity in the static limit. This singularity can only be regularized by introducing a nonperturbative magnetic screening mass in the calculation of the damping rate of a fast parton.

We can use the definition of parton interaction rates,

$$
\begin{aligned}
\Gamma_{\text {loss }}\left(p_{1}\right) & =\frac{\nu_{2}}{(2 \pi)^{5}} \int d^{3} p_{2} d^{3} q d \omega f_{2}\left(1 \pm f_{3}\right)\left(1 \pm f_{4}\right) \frac{1}{2}\left|M_{12 \rightarrow 34}\right|^{2} \delta\left(\omega-\mathbf{v}_{\mathbf{1}} \cdot \mathbf{q}\right) \delta\left(\omega-\mathbf{\mathbf { v } _ { 2 }} \cdot(\mathbf{q}) 1\right) \\
\Gamma_{\text {gain }}\left(p_{1}\right) & =\frac{\nu_{2}}{(2 \pi)^{5}} \int d^{3} p_{2} d^{3} q d \omega f_{3} f_{4}\left(1 \pm f_{2}\right) \frac{1}{2}\left|M_{12 \rightarrow 34}\right|^{2} \delta\left(\omega-\mathbf{v}_{\mathbf{1}} \cdot \mathbf{q}\right) \delta\left(\omega-\mathbf{v}_{\mathbf{2}} \cdot \mathbf{q}\right),
\end{aligned}
$$

and rewrite the Boltzmann equation as

$$
\begin{aligned}
v_{1} \cdot \partial f_{1} & =-f_{1} \Gamma_{\text {loss }}\left(p_{1}\right)+\left(1 \pm f_{1}\right) \Gamma_{\text {gain }}\left(p_{1}\right) \\
& =-\Gamma\left(p_{1}\right)\left(f_{1}-\tilde{f}\right)
\end{aligned}
$$

where

$$
\Gamma(p)=\Gamma_{\text {loss }}(p) \mp \Gamma_{\text {gain }}(p)
$$

is usually referred to as the damping rate of a particle (or a quasiparticle), and $\tilde{f}$ is defined as

$$
\tilde{f}(p)=\frac{\Gamma_{\text {gain }}(p)}{\Gamma_{\text {loss }}(p) \mp \Gamma_{\text {gain }}(p)} .
$$


For a system in local thermal equilibrium, one can relate the damping rate to the imaginary part of the gluon self-energy [22,

$$
\operatorname{Im}_{\mu}^{\mu}(p)=-2(p \cdot u) \Gamma(p),
$$

where the factor 2 comes from our definition of the interaction rates among identical particles. One can also show that, if $f(p)$ takes the local equilibrium form $f^{\mathrm{eq}}(p)=$ $(\exp (p \cdot u / T) \mp 1)^{-1}$

$$
\frac{\Gamma_{\text {loss }}(p)}{\Gamma_{\text {gain }}(p)}=e^{p \cdot u / T}
$$

using the energy and momentum conservation and the identity $1 \pm f^{\mathrm{eq}}(p)=f^{\mathrm{eq}}(p) \exp (p$ $u / T)$. Therefore, by definition, $\tilde{f}(p)$ becomes $f^{\mathrm{eq}}(p)$. Thus, the global equilibrium distribution $f^{\mathrm{eq}}(p)$ is a solution to the Boltzmann equation if the flow velocity $u$ is independent of space and time.

We can complete the angular integrations in Eqs. (11) and (12). Making approximations $f\left(p_{3}\right) \approx f\left(p_{1}\right)$ and $f\left(p_{4}\right) \approx f\left(p_{2}\right)$, we can also complete the integration over $p_{2}$ by cutting off the integration over $q$ at $q_{\max } \approx 3 T$ [17]. We then obtain the gluon damping rate,

$$
\Gamma^{g g}=\frac{g^{4}}{4 \pi} \frac{C_{g g} \nu_{g}}{12} T^{3} \int_{-1}^{1} d x \int_{0}^{q_{\max }^{2}} d q^{2}\left\{\frac{1}{\left|q^{2}+\pi_{L}(x)\right|^{2}}+\frac{1}{2} \frac{\left(1-x^{2}\right)^{2}}{\left|q^{2}\left(1-x^{2}\right)+\pi_{T}(x)\right|^{2}}\right\},
$$

including only gluon-gluon scatterings.

The contribution from longitudinal interactions is finite and proportional to $g^{2} T$ due to the Debye screening. However, Debye screening is absent in the transverse interactions in the static limit. There is a logarithmic divergency even if Landau damping is taken into account. One solution to this problem is to add a nonperturbative magnetic mass $m_{m a g} \sim g^{2} T$ to the transverse self-energy $\pi_{T}(x)$. In this case, the dominant contribution of the transverse interactions comes from $m_{\operatorname{mag}} \lesssim q \lesssim q_{D}$ and thus is proportional to $g^{2} T \ln \left(q_{D} / m_{\mathrm{mag}}\right)$ which is independent of $q_{\max } \gg q_{D}$. With $\pi_{L}(x)$ and $\pi_{T}(x)$ given by Eqs. (9) and (10), we can complete the numerical integration. A fit to the numerical result gives us

$$
\Gamma^{g g}=\frac{g^{4}}{4 \pi} \frac{C_{g g} \nu_{g}}{6} \frac{T^{3}}{q_{D}^{2}}\left[\left(\ln \frac{q_{D}^{2}}{m_{\mathrm{mag}}^{2}}-1.0+2.0 \frac{m_{\mathrm{mag}}^{2}}{q_{D}^{2}}-0.32 \frac{q_{D}^{2}}{q_{\max }^{2}}\right)+1.1 \frac{q_{\max }^{2}}{q_{\max }^{2}+q_{D}^{2}}\right],
$$

where the first term comes from the transverse interactions while the second from the longitudinal ones. Using the estimate of $m_{\text {mag }} \approx 0.255 \sqrt{N_{c} / 2} g^{2} T$ from Ref. [23 and neglecting the quark contribution to the Debye screening mass, we have

$$
\Gamma^{g g} \approx N_{c} \alpha_{s} T\left[\ln \left(1 / \alpha_{s}\right)-0.1+\mathcal{O}\left(\alpha_{s}\right)\right]
$$

Note that contributions to the order $\alpha_{s}^{2}$ in Eq. (19) have been neglected, since they are not complete in our calculation. In order to have a complete calculation of such 
higher order corrections, one has to include thermal vertex and vacuum corrections which should depend on the renormalization scale. The final result to this order should be invariant under the renormalization group. This result agrees with previous calculations [11, 12, 13] to the leading order of $\alpha_{s}$ which depends only on the imaginary part of the transverse self-energy, $\pi_{T}(x) \approx-i(\pi / 4) q_{D}^{2} x$ at small $x$. Inclusion of the full expression of the self-energy only contribute to the next order corrections. The increase in scattering by including quarks is exactly compensated by the increase in Debye screening due to quarks [12].

For a soft gluon $(p \sim g T)$, one can not neglect its thermal mass anymore. The damping rate for a gluon at rest will not have the logarithmic divergency in the transverse interaction, since the exchanged gluon must carry nonzero momentum and energy at least of order of $g T$ and thus never approach to the static limit. In addition the transverse (magnetic) interactions are reduced by velocity factors which for the massive partons are smaller than the speed of light. The damping rate in this case was found by Braaten and Pisarski [10] to the leading order as

$$
\Gamma^{g g}(0) \approx 1.1 N_{c} \alpha_{s} T
$$

Apparently, the damping rate has a nontrivial momentum dependence [24].

As we have mentioned, $f^{\mathrm{eq}}(p \cdot u / T)$ is a solution to the Boltzmann equation as far as the flow velocity is uniform in space and time. We should emphasize here that the damping rate does not determine how rapidly a system near equilibrium approaches it as one would naively think. The thermalization time is actually related to the transport rates [14, 15, 16, 17, 18]. The easiest way to prove this is to check that the logarithmic divergency that has plagued the calculation of the damping rate of a fast gluon does not appear in the Boltzmann equation. To check this, we make the following expansion:

$$
f\left(p_{3,4}\right) \approx f\left(p_{1,2}\right) \pm \omega f^{\prime}\left(p_{1,2}\right)+\frac{\omega^{2}}{2} f^{\prime \prime}\left(p_{1,2}\right)
$$

for small angle scatterings. The function $F_{1234}[f]$ in Boltzmann equation becomes

$$
F_{1234}[f]=-\frac{q^{2} x^{2}}{2}\left[f_{1}\left(1 \pm f_{1}\right) f_{2}^{\prime \prime}+f_{2}\left(1 \pm f_{2}\right) f_{1}^{\prime \prime}-2\left(1 \pm f_{1} \pm f_{2}\right) f_{1}^{\prime} f_{2}^{\prime}\right]
$$

which is proportional to $q^{2}$. Here we have dropped terms linear in $x$ since they vanish after integration over $x$. One can verify that this function after expansion still vanishes for the equilibrium distribution, $F_{1234}\left[f^{\text {eq }}\right]=0$. For a system away from equilibrium, the collisional integral in the Boltzmann equation is nonzero but finite despite the logarithmic singularity in the transverse part of the matrix element squared, $\left|M_{12 \rightarrow 34}\right|^{2}$ when $q, \omega \rightarrow 0$. Because of the factor $q^{2}$ in $F_{1234}[f]$, Landau damping in the self-energy of the exchanged gluon is sufficient to give a finite value of the collisional integral. In other words, thermalization not only depends on the parton interaction rates but also 
on the efficiency of transferring momentum in each interaction. Those interactions with zero energy and momentum transfers do not contribute to the thermalization process, though their cross sections are infinitely large. This is why the thermalization time and other transport coefficients do not suffer from the infrared divergency as pointed out in a number of papers 12, 16, 17, 18.

For a system near equilibrium, one can characterize the deviation from equilibrium by $\delta f=f-f^{\text {eq }}=-\theta(p) p \cdot \partial f /(p \cdot u)$ in a relaxation time approximation. Equivalently, one has

$$
\frac{1}{p \cdot u} p \cdot \partial f=-\frac{f-f^{\mathrm{eq}}}{\theta(p)} .
$$

In general the relaxation time $\theta(p)$ depends on momentum $p$ and in principle can be obtained by solving the linearized Boltzmann equation. In this paper, we neglect the momentum dependence of the relaxation time. For a pure gluonic gas near local thermal equilibrium where thermalization is achieved through viscous relaxation, the relaxation time is (Appendix B)

$$
\frac{1}{\theta} \simeq 0.92 N_{c}^{2} T \alpha_{s}^{2} \ln \left(\frac{1.6}{N_{c} \alpha_{s}}\right) .
$$

Again, because of the extra factor $q^{2}$ appearing in the transport rate, the Debye screening and Landau damping are sufficient to regularize the effective transport cross section. The dominant contribution comes from interactions with $q_{D} \lesssim q \lesssim q_{\max }$, leading to a logarithmic factor $\ln \left(q_{\max } / q_{D}\right)$ as compared to $\ln \left(q_{D} / m_{\text {mag }}\right)$ in the gluon damping rate. Therefore, the dependence of the relaxation time on the (weak) coupling constant and the color dimension $N_{c}$ is quite different from the gluon damping rate.

If the system is close to thermal equilibrium, the hydrodynamic equations from energy-momentum conservation to the zeroth order of $\delta f$ can give us the time evolution of the temperature $T$. For an ideal gluon gas with one-dimensional expansion, $T$ decreases like $T / T_{0}=\left(\tau_{0} / \tau\right)^{1 / 3}$. Therefore, the relaxation time $\theta$ increases with time with a power of $1 / 3$,

$$
\theta=\frac{\left(\tau / \tau_{0}\right)^{1 / 3}}{0.92 N_{c}^{2} T_{0} \alpha_{s}^{2} \ln \left(1.6 / N_{c} \alpha_{s}\right)} .
$$

A more general time dependence of the relaxation time can have a power-law form,

$$
\theta=\theta_{0}\left(\tau / \tau_{0}\right)^{\beta},
$$

which also covers both the constant $(\beta=0)$ and the linear $(\beta=1)$ cases as have been studied by several authors [25, 26, 27, 28]. The latter case arises when a constant scattering cross section, $\sigma$, is assumed for the relaxation time, $\theta \sim(\sigma n)^{-1}$, and with a density decreasing as $n \sim \tau^{-1}$ due to one-dimensional expansion. For a system far away from thermal equilibrium, the time dependence may differ from Eq. (26). Our earlier calculations [8] show that an initially free-streaming system has only 
a logarithmic time dependence. This is because the phase space for small angle scatterings $\left(q \sim q_{D}\right)$ opens up quadratically with time in the free-streaming case and it balances the decrease in parton density.

We would like to emphasize that the weak time dependence of the relaxation time in Eq. (26) depends very sensitively on the Debye screening of the small-angle parton scatterings which restricts the momentum transfer to $q \gtrsim q_{D}=g T$. Smaller Debye screening mass due to the decrease of the temperature, gives a larger interaction rate which then compensates the decrease of the parton density and thus gives the weak time dependence of the relaxation time. If we use a fixed momentum cut-off $q_{\text {cut }}$, as in most of the numerical simulations of parton production [3] and cascade [4], instead of a time dependent Debye screening mass, the effective transport cross section will remain constant, proportional to $N_{c}^{2} \alpha_{s}^{2} / T^{2} \ln \left(T^{2} / q_{\text {cut }}^{2}\right)$. The resultant relaxation time

$$
\frac{1}{\theta} \propto N_{c}^{2} \alpha_{s}^{2} T \ln \left(T / q_{\text {cut }}\right)
$$

will increase more rapidly with time.

If we have to include transverse expansion later in the evolution of a system, then the temperature will decrease faster, like $\tau_{0} / \tau$, than in the one-dimensional case assuming hydrodynamic expansion. The relaxation time even with the inclusion of Debye screening will increase linearly with time. The relaxation time with a momentum cut-off applied to parton interactions will increase faster than linear with time, which will only lead the system into free-streaming.

At this point we should emphasize that we have only considered the lowest order contribution from $2 \leftrightarrow 2$ processes in our calculation of the relaxation time. In principle, higher order processes, like $2 \leftrightarrow 2+n$, should also contribute to the thermalization. Such processes can be included by considering high order thermal vertex corrections. For a complete calculation, one should also include vacuum corrections and the result should depend on the renormalization scale and obey the renormalization group equation. In general, contributions from $2 \leftrightarrow 2+n$ processes should have a form 29,

$$
\Gamma_{n} \sim \Gamma_{0}\left[\alpha_{s} \ln \left(q^{2} / q_{0}^{2}\right)\right]^{n}
$$

where $q^{2}$ is the momentum scale of these processes. At zero temperature, $q_{0}$ is some confinement scale below which perturbative QCD is no longer applicable. At finite temperature, $q_{0}$ is very likely to be replaced by screening masses. Since the largest momentum scale in a system at finite temperature is $q^{2} \sim T^{2}$, the leading correction from $2 \leftrightarrow 2+n$ processes must be,

$$
\Gamma_{n} \sim \Gamma_{0}\left[\alpha_{s} \ln \left(1 / \alpha_{s}\right)\right]^{n}
$$

Such corrections therefore are high orders in $\alpha_{s} \ln \left(1 / \alpha_{s}\right)$ and are negligible in the week couple limit. For temperatures not far above the QCD phase transition temperature 
$T_{c} \sim 200 \mathrm{MeV}$, the strong coupling constant is not very small. The above contributions might not be negligible. However, for an order-of-magnitude estimate, we can neglect these higher order contributions. If one considers the chemical equilibration of a kinetically thermalized system as in Ref. [6], $n \leftrightarrow m$ multiplication processes become very important. The leading contribution to the chemical equilibration, in this case, comes from $2 \leftrightarrow 3$ processes.

\section{Approach to thermal equilibrium}

Let us consider the early stage of a very heavy ion collision where transverse expansion is not important yet. We then can treat the system as a one-dimensional system. We assume along with Bjorken [30] a scaling flow velocity

$$
u_{\mu}=\frac{x_{\mu}}{\tau}=\left(\cosh \eta, \sinh \eta, \mathbf{0}_{\perp}\right)
$$

in the longitudinal direction, where

$$
\tau=\sqrt{t^{2}-z^{2}}, \quad \eta=\frac{1}{2} \ln \left(\frac{t+z}{t-z}\right)
$$

are the proper time and spatial rapidity, respectively. In terms of these new variables, the Boltzmann equation in the relaxation time approximation for a system near thermal equilibrium becomes

$$
\frac{\partial f}{\partial \tau}-\frac{\tanh \xi}{\tau} \frac{\partial f}{\partial \xi}=-\frac{f-f^{\mathrm{eq}}}{\theta}
$$

where $\xi=\eta-y$ and $y$ is the rapidity of a particle,

$$
y=\frac{1}{2} \ln \left(\frac{E+p_{z}}{E-p_{z}}\right) .
$$

Since $p \cdot u=p_{T} \cosh \xi$, we can see that the solution to the above Boltzmann equation is a function of $\xi, \tau$ and $p_{T}$, and is Lorentz invariant under longitudinal boost. One may then, as done in [25], solve the Boltzmann equation in the central slice only, i.e., $\eta=0$. To simplify the problem, we assume the system is already in chemical equilibrium so that the gluon chemical potential vanishes. This is not always true in a realistic situation as shown by numerical simulations [5] of initial parton production at around RHIC energies. However, at LHC energies, the small- $x$ behavior of the parton distributions as measured by recent HERA experiments [31] gives much higher densities of initially produced partons very close to chemical equilibrium 32]. For a given momentum and time dependence of the relaxation time, one can find the solution to Eq. (33) in an integral form,

$$
\begin{aligned}
f\left(p_{T}, \xi, \tau\right) & =e^{\chi_{0}-\chi} f_{0}\left(p_{T}, \sinh ^{-1}\left(\frac{\tau \sinh \xi}{\tau_{0}}\right)\right) \\
& +\int_{\chi_{0}}^{\chi} d \chi^{\prime} e^{\chi-\chi^{\prime}} f^{\mathrm{eq}}\left(p_{T}, \sinh ^{-1}\left(\frac{\tau \sinh \xi}{\tau^{\prime}}\right), T^{\prime}\right)
\end{aligned}
$$


Here $f_{0}\left(p_{T}, \xi\right)$ is the initial distribution at time $\tau_{0}$, and the time dependence of the temperature in $f^{\mathrm{eq}}\left(p_{T}, \xi, T\right)$ is determined by requiring the energy density for $f$ and $f^{\text {eq }}$ be equal at any time,

$$
\epsilon(T) \equiv \int d p E f(p)=\int d p E f^{\mathrm{eq}}(p, T) .
$$

The variable $\chi$ is defined by

$$
\chi\left(p_{T}, \xi, \tau\right)=\chi_{0}+\int_{\tau_{0}}^{\tau} d \tau^{\prime} \theta^{-1}\left(p_{T}, \sinh ^{-1}\left(\frac{\tau \sinh \xi}{\tau^{\prime}}\right), \tau^{\prime}\right) .
$$

If we take the form in Eq. (27) for a momentum-independent relaxation time, then

$$
\chi=\chi_{0}\left(\frac{\tau}{\tau_{0}}\right)^{1-\beta}, \quad \chi_{0}=\frac{\tau_{0} / \theta_{0}}{1-\beta},
$$

for $\beta \neq 1$. The case for $\beta=1$ was studied by Gavin 28. For a more general discussion, let us also assume a initial parton distribution

$$
\frac{d N}{d \xi d p_{T}^{2}}=w\left(p_{T}\right) \theta(|\xi|-Y)
$$

with a simple plateau distribution in $\xi$. One can also consider a gaussian form of distribution [34], but the above form is simpler. The width $Y$ is a measure of the initial correlation between space and momentum, or $\eta$ and $y$. This width which normally depends on the transverse momentum $p_{T}$ [33, 34] can be estimated by the uncertainty principle. We will assume it a constant for simplicity and will study how the thermalization process depends on the initial condition by varying $Y$. Here, $w\left(p_{T}\right)$ is the $p_{T}$ distribution which usually has a power-law or exponential form. The corresponding phase space density within a volume $V$ is

$$
f_{0}\left(p_{T}, \xi\right)=\frac{2(2 \pi)^{2}}{\nu_{g} V} \frac{w\left(p_{T}\right)}{p_{T} \cosh \xi} \theta(|\xi|-Y)
$$

with an initial energy density

$$
\epsilon_{0}=\frac{2 \sinh Y}{V} \int d p_{T}^{2} p_{T} w\left(p_{T}\right)
$$

Following Baym [25], we study the solution to the Boltzmann equation by taking the first moment (energy density) of Eq. (35) with respect to the single parton energy. Defining,

$$
G=\frac{\tau \epsilon(\tau)}{\tau_{0} \epsilon_{0}}
$$

we have from Eq. (35)

$$
G(\chi)=e^{\chi_{0}-\chi} H_{Y}\left(\left(\chi_{0} / \chi\right)^{\frac{1}{1-\beta}}\right)+\int_{\chi_{0}}^{\chi} d \chi^{\prime} e^{\chi^{\prime}-\chi} G\left(\chi^{\prime}\right) h\left(\left(\chi^{\prime} / \chi\right)^{\frac{1}{1-\beta}}\right)
$$


where $\chi^{\prime} / \chi=\left(\tau^{\prime} / \tau\right)^{1-\beta}$ from Eq. (38), and

$$
\begin{aligned}
H_{Y}(a) & =\frac{1}{\sinh Y} \int_{0}^{Y} d \xi \sqrt{1+a^{2} \sinh ^{2} \xi}, \\
h(a) & =\frac{1}{2}\left(a+\frac{\arcsin \sqrt{1-a^{2}}}{\sqrt{1-a^{2}}}\right) .
\end{aligned}
$$

If the initial distribution $f_{0}$ is an equilibrium one, one should replace $H_{Y}(a)$ by $h(a)$ in Eq. (43). However, $H_{Y}(a)$ [with $H_{Y}(1)=1$ and $H_{Y}(0)=Y / \sinh Y$ ] covers a wide selection of initial distributions with different values of $Y$. For $Y=1.23$ (i.e., $Y / \sinh Y=h(0)=\pi / 4), H_{Y}(a)$ is very similar to $h(a)$ for an isotropic initial distribution. When $Y=0$, the initial distribution corresponds to the Bjorken scaling ansatz $(\eta=y)$. One then has $H_{0}(a)=1$, which is similar to the case discussed by Baym [25]. After performing a partial integration in Eq. (43), we have finally

$$
\int_{\chi_{0}}^{\chi} d \chi^{\prime} e^{\chi^{\prime}} \frac{d}{d \chi^{\prime}}\left[G\left(\chi^{\prime}\right) h\left(\left(\chi^{\prime} / \chi\right)^{\frac{1}{1-\beta}}\right)\right]=e^{\chi_{0}}\left[H_{Y}\left(\tau_{0} / \tau\right)-h\left(\tau_{0} / \tau\right)\right] .
$$

The discussion of the condition for equilibrium at large times, $\tau$, is now similar to our earlier analysis [8] even with the general initial condition assumed. However, as we will show later, the initial condition will have important influences on the approach to equilibrium and the final entropy production.

For $\beta<1, \tau \rightarrow \infty$ corresponds to $\chi \rightarrow \infty$. In this limit, the r.h.s. of Eq. (46) is a finite number, $e^{\chi_{0}}(Y / \sinh Y-\pi / 4)$. Since the integrand on the 1.h.s. of Eq. (46) has an exponential factor, a finite integral must require

$$
\frac{d}{d \chi^{\prime}}\left[G\left(\chi^{\prime}\right) h\left(\left(\chi^{\prime} / \chi\right)^{\frac{1}{1-\beta}}\right)\right]_{\chi^{\prime}=\chi}=0, \quad \chi \rightarrow \infty .
$$

Using $h(1)=1$ and $h^{\prime}(1)=1 / 3$, one can find the solution to the above equation,

$$
G \propto \chi^{\frac{-1}{3(1-\beta)}} \propto\left(\tau_{0} / \tau\right)^{1 / 3}, \quad \tau / \tau_{0} \rightarrow \infty
$$

which corresponds to the one-dimensional hydrodynamic limit, $\epsilon \propto\left(\tau_{0} / \tau\right)^{4 / 3}$. Therefore, thermal equilibrium will eventually be established for $\beta<1$.

For $\beta>1, \tau \rightarrow \infty$ limit corresponds to $\chi \rightarrow-0$. Using $h(0)=\pi / 4$, one has from Eq. (43)

$$
G(\chi)=e^{\chi_{0}}-\frac{\pi}{4} \int_{0}^{\chi_{0}} d \chi^{\prime} G\left(\chi^{\prime}\right)=\text { const. }, \quad \chi \rightarrow-0 .
$$

This corresponds to the free-streaming limit, $\epsilon \propto \tau_{0} / \tau$. Therefore, thermal equilibrium will never be achieved if $\beta>1$.

The special case $\beta=1$ was studied by Gavin [28] who found that the system will also reach an asymptotic state lying in between free-streaming and hydrodynamic limit depending on the value of the prefactor $\theta_{0}$ in Eq. (27). In the asymptotic state, $G\left(\tau / \tau_{0}\right) \propto\left(\tau_{0} / \tau\right)^{\gamma}$ with $0 \leq \gamma<1 / 3$. Only for a very large $\theta_{0} / \tau_{0} \gg 1$, does the 


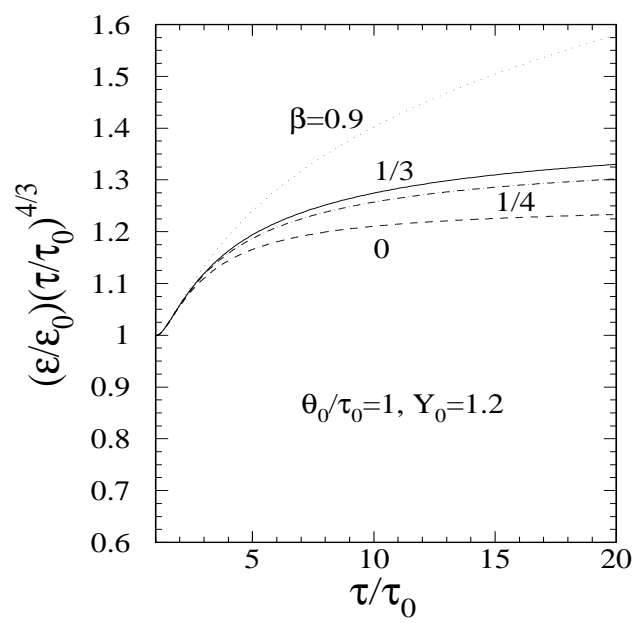

Fig. 1

Figure 1: Time evolution of $G\left(\tau / \tau_{0}\right)\left(\tau / \tau_{0}\right)^{1 / 3}=\left(\epsilon / \epsilon_{0}\right)\left(\tau / \tau_{0}\right)^{4 / 3}$ according to the solution to Boltzmann equation, for different time dependence of the relaxation time $\theta=\theta_{0}\left(\tau / \tau_{0}\right)^{\beta}$, with $\theta_{0} / \tau_{0}=1, \beta=0.9$ (dotted), $1 / 3$ (solid), $1 / 4$ (dot-dashed)and 0 (dashed line). $Y_{0}=1.2$ is the width of the initial rapidity distribution.

system approach to the hydrodynamic limit with $\gamma \approx 1 / 3-16 \tau_{0} / 45 \theta_{0}$, which is very close in form to the case of $\beta \lesssim 1$ as noted by Gavin in Ref. [28].

Shown in Figs. 1 and 2 are the numerical solutions of Eq. (43) for different relaxation times. For a clear presentation, we plot $\left(\tau / \tau_{0}\right)^{1 / 3} G\left(\tau / \tau_{0}\right)=\left(\tau / \tau_{0}\right)^{4 / 3}\left(\epsilon / \epsilon_{0}\right)$ as a function of $\tau / \tau_{0}$ for different values of $\beta$ and $\theta_{0} / \tau_{0}$. In these two plots, we have chosen $Y=1.2$ which corresponds to an isotropic initial distribution in momentum space. If the system undergoes free streaming, i.e., $G\left(\tau / \tau_{0}\right)=$ const., the plotted quantity should increase with $\tau / \tau_{0}$ with a power of $1 / 3$. Thus we see from Figs. 1 and 2 that a system must undergo free streaming for a period of time before it approaches the equilibrium limit when parton interactions eventually balance the expansion. The duration of such a period, which we can define as the thermalization time $\tau_{\text {th }}$ is determined by the relaxation time and its time dependence. For fixed $\beta$, the thermalization time $\tau_{\text {th }}$ is approximately proportional to $\theta_{0}$. From Fig. 2, we can estimate that $\tau_{\text {th }} \simeq 5 \theta_{0}$ for $\beta=1 / 3$ if we consider that thermalization is reached when $G\left(\tau / \tau_{0}\right)$ is about $10 \%$ from its hydrodynamical limit. Assuming one scattering is sufficient to thermalize the system [35] is therefore a serious underestimate. When $\beta$ is close to 1 , the system approaches the hydrodynamical limit very slowly as seen in Fig. 1. However, the hydrodynamical limit is still achieved as long as $\beta<1$, unlike when $\beta \geq 1$. When the thermalization time is very long, one must also consider whether 3-dimensional expansion and/or hadronization occur earlier. 


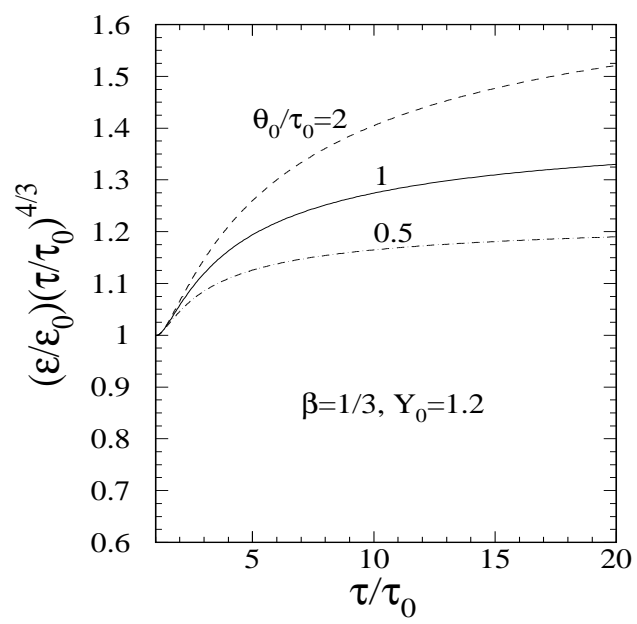

Fig. 2

Figure 2: Same as Fig. (四), expect for $\beta=1 / 3, \theta_{0} / \tau_{0}=2$ (dashed), 1 (solid) and 0.5 (dot-dashed line).

\section{Memory effect}

Let us now discuss how the initial condition $f_{0}$ influences the thermalization process and the final entropy production. In Fig. 3 the time evolution of $\left(\tau / \tau_{0}\right)^{1 / 3} G\left(\tau / \tau_{0}\right)$ is shown for different values of $Y$ which characterizes the initial distribution of partons in the phase space. For large values of $Y$, we notice that the system initially expands even faster than the ideal hydrodynamical case and then turns over, approaching the hydrodynamical limit. To understand this, let us take the first moment of Eq. (33) with respect to the single parton energy. We then have, by energy and momentum conservation,

$$
\frac{d \epsilon}{d \tau}+\frac{\epsilon+P_{L}}{\tau}=0
$$

where the energy density is defined by Eq. (36) and

$$
P_{L}=\nu_{g} \int \frac{d^{3} p}{(2 \pi)^{3}} \frac{p_{z}^{2}}{E} f(p)
$$

is the longitudinal pressure. The solution to the Boltzmann equation can be parametrized as $P_{L}(\tau)=\gamma(\tau) \epsilon(\tau)$ with $0<\gamma<1$. We have then from Eqs. (50) and (42),

$$
G(\tau)=e^{-\int_{\tau_{0}}^{\tau} d \tau^{\prime} \frac{\gamma\left(\tau^{\prime}\right)}{\tau^{\prime}}}
$$

Thus, $\gamma(\tau)$ characterizes the thermalization of the system (or equal partition in longitudinal and transverse direction). In the hydrodynamical limit, $\gamma=1 / 3$, while 


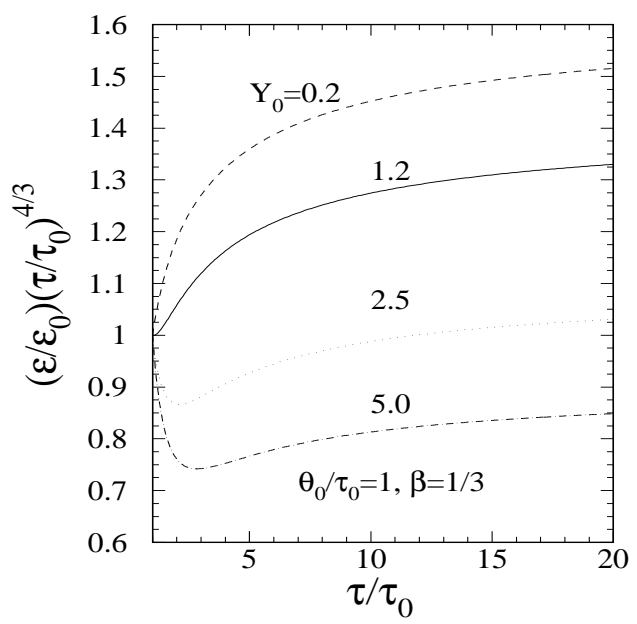

Fig. 3

Figure 3: Same as Fig. (11), except for $\beta=1 / 3, \theta_{0} / \tau_{0}=1, Y_{0}=0.2$ (dashed), 1.2 (solid), 2.5 (dotted) and 5.0 (dot-dashed line).

free-streaming corresponds to $\gamma=0$. One can expect that the initial evolution of the system near $\tau / \tau_{0} \sim 1$ should be determined by the value of $\gamma_{0}=\gamma\left(\tau_{0}\right)$,

$$
G(\tau) \simeq\left(\tau_{0} / \tau\right)^{\gamma_{0}}, \quad \tau / \tau_{0} \sim 1
$$

Using the initial distribution in Eq. (40), we have

$$
\gamma_{0}=\frac{P_{L}\left(\tau_{0}\right)}{\epsilon_{0}}=1-\frac{\arctan (\sinh Y)}{\sinh Y} .
$$

An isotropic situation corresponds to $\arctan \left(\sinh Y_{0}\right) / \sinh Y_{0}=2 / 3$ or $Y_{0}=1.167$ which is very close to the value we obtained by requiring $H_{Y}(0)=\pi / 4$. For $Y<Y_{0}$, $\gamma_{0}<1 / 3$, the system starts its evolution more like free streaming as we have noticed in our numerical solutions. The extreme case is the Bjorken scaling ansatz, $Y=0$, $\gamma_{0}=0$, which corresponds exactly to free streaming. For $Y>Y_{0}, \gamma_{0}>1 / 3$, the system will initially expand in the longitudinal direction even faster than a thermal expansion, as also demonstrated in our numerical solutions. Physically, this is caused by the higher longitudinal pressure built up by the large amount of partons which are distributed over a large range of rapidity in the local frame. One can check that the expansion in this early stage is still dominated by free-streaming. However, working against such high pressure costs energy thus leading to less entropy production as we now show in the following.

Since the system will eventually approach the thermal equilibrium limit when $\beta<1$, we can define a prefactor $\mathcal{A}_{\infty}$ by

$$
G\left(\tau / \tau_{0}\right)=\mathcal{A}_{\infty}\left(\tau_{0} / \tau\right)^{1 / 3}, \quad \tau / \tau_{0} \rightarrow \infty
$$




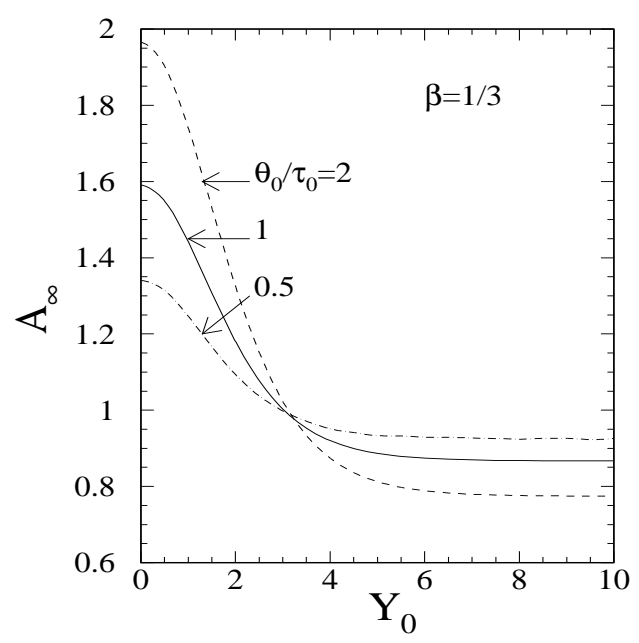

Fig. 4

Figure 4: The prefactor, $\mathcal{A}_{\infty}=\left(\epsilon / \epsilon_{0}\right)\left(\tau / \tau_{0}\right)^{4 / 3}\left(\tau / \tau_{0} \rightarrow \infty\right)$, as a function of the $Y_{0}$, the width of the initial rapidity distribution, for different relaxation times $\theta=\theta_{0}\left(\tau / \tau_{0}\right)^{\beta}$ $(\beta=1 / 3), \theta_{0} / \tau_{0}=2$ (dashed), 1 (solid), and 0.5 (dot-dashed line).

This prefactor in general can only be calculated numerically and will depend on the relaxation time as well as the initial condition as we have seen in Fig. 3. Using the above expression, one can calculate the final entropy of the system per comoving volume 8

$$
\tau s_{f}=\frac{4}{3} \frac{\tau_{0} \epsilon_{0}}{T_{0}} \frac{T_{0}}{T} G\left(\tau / \tau_{0}\right)=\tau_{0} \frac{4}{3} \frac{\epsilon_{0}}{T_{0}} \mathcal{A}_{\infty} .
$$

which is exactly proportional to $\mathcal{A}_{\infty}$. Here $T_{0}$ is only a parameter in the time dependence of the final temperature, $T=T_{0}\left(\tau_{0} / \tau\right)^{1 / 3}$. Since the initial system is not in equilibrium especially for large values of $Y,(4 / 3) \epsilon_{0} / T_{0}$ should not be considered as the initial entropy density. Therefore, $\mathcal{A}_{\infty}$ is not the absolute increase of the total entropy over its initial value, except when the initial distribution is an equilibrium one. Nevertheless, $\mathcal{A}_{\infty}$ still carries a lot of information about the thermalization process of the system. We plot this prefactor in Fig. 1 as a function of $Y$ for $\beta=1 / 3$ but for different values of $\theta_{0} / \tau_{0}$. It is clear that there is less entropy production for larger values of $Y$, since the system has to work against increasingly high longitudinal pressure thus converting its kinetic energy to expansion energy. The system has the maximum entropy production when the initial distribution is that of the Bjorken scaling ansatz, $Y=0$. In this case, one can calculate [8] that the entropy production increases with the relaxation time like $\mathcal{A}_{\infty} \propto\left(\theta_{0} / \tau_{0}\right)^{1 / 4(1-\beta)}$. For very large values of $Y, \mathcal{A}_{\infty}$ decreases slightly with $\theta_{0} / \tau_{0}$ as indicated in Fig. 1 , since the system has to spend longer time work against the extraordinaryly high longitudinal pressure. It is the competition between long thermalization time (thus entropy production) and 
long duration of work against high pressure that reverses the $\theta_{0} / \tau_{0}$ dependence of entropy production $\mathcal{A}_{\infty}$.

\section{Conclusion and outlook}

In this paper, we investigated the thermalization process in a one-dimensional expanding parton plasma within the framework of the Boltzmann equation. In particular, we have studied the time dependence of the relaxation time and its influence on the thermalization. If the time dependence is weaker than a linear form, we find that the thermal equilibrium limit will eventually be reached. For a time dependence stronger than the linear one, the system will never thermalize, only leading to a free-stream limit. For an exact linear time dependence, the system will reach an asymptotic state between free-streaming and thermal equilibrium. We find that the thermalization process also depends on the initial condition of the system. The deviation of the initial momentum distribution from an isotropic one in the longitudinal direction determines the initial approach to thermal equilibrium. This initial approach will then carry its inertia throughout the whole thermalization process. This "memory effect" can be seen from the dependence of the final total entropy production on the initial momentum distributions.

Using perturbative QCD at finite temperature in the transport theory, we have calculated the relaxation time as a result of parton scatterings. We have pointed out the important differences between parton damping rates and thermalization times. To regularize the singular behavior of the parton scattering cross sections, we have used a full gluon propagator which includes the resummation of an infinite number of hot thermal loops. Because of the singular behavior of the parton scattering matrix elements, the resultant relaxation time depends sensitively on the Debye screening mass $q_{D}=g T$. In an expanding parton gas, the temperature decreases with time and so does the Debye screening mass, thus leading to an increasing transport cross section. This then compensates the decrease of the parton density and gives us a relaxation time with a weak time dependence, $\theta \propto\left(\tau / \tau_{0}\right)^{1 / 3}$. However, if one introduces a fixed momentum cut-off to parton scatterings as in most of numerical simulations, one will introduce an extra logarithmic time dependence which will slow down the thermalization process.

Although we have demonstrated the necessity of the inclusion of Debye screening and Landau damping in the study of the parton thermalization in an expanding system, it is not clear to us how to incorporate them into numerical simulations such as parton cascade models. One can include the Debye screening semiclassically 19 by brutal force. However, one immediate problem we have to solve is how to avoid double counting.

Since we used the full propagator which includes many thermal loops, we have 
effectively included multiple particle scatterings. One can easily see this by expanding the full propagator in terms of the bare propagators at zero temperature and the selfenergy from thermal loops. The contribution from the real part of the self-energy (mainly Debye screening) corresponds to multiple particle scatterings in the thermal bath. One can in principle include particle radiation and absorption by considering the thermal loop corrections to the full vertices. In doing so, one can automatically avoid both the infrared and collinear divergencies [36] which one normally regularizes by resorting to two additional cut-offs [37].

As we have demonstrated in Appendix B, the imaginary part of the self-energy also contributes to the effective parton scattering in a parton gas. In fact, the imaginary part, which is responsible for Landau damping, is necessary to regularize the transverse interaction since there is no magnetic screening in QCD. The contribution from this imaginary part to an effective two-parton scattering corresponds to independent scatterings of the two partons off particles in the thermal bath. This can easily cause double counting in a parton cascade model because independent parton scatterings are also simulated over the volume of the system. One might be able to avoid this problem by introducing a length scale in the order of $1 / g T$, within which multiple particle scatterings are not allowed and only one effective two-parton scattering (with Debye screening and Landau damping) is permitted.

\section{Acknowledgement}

This work was supported by the Director, Office of Energy Research, Office of High Energy and Nuclear Physics, Division of Nuclear Physics, of the U.S. Department of Energy under Contract DE-AC03-76SF00098, and the Danish Natural Science Research Council. Discussions with V. Koch are gratefully acknowledged.

\section{Appendices}

\section{A Effective matrix elements of forward parton scat- terings}

In this appendix we separate the QCD interactions into longitudinal and transverse parts with inclusion of screening in these two parts and show the simplifications that appear in the limit of forward scatterings or small momentum transfer interactions. We adopt the same convention as in Ref. [21] and split a vector $Q_{\mu}$ into its components parallel and orthogonal to the flow velocity $u_{\mu},\left(u^{\mu} u_{\mu}=1\right)$, such that

$$
\omega=Q \cdot u, \quad \tilde{Q}_{\mu}=Q_{\mu}-u_{\mu}(Q \cdot u)
$$


We can then denote a vector by $Q=[\omega, \mathbf{q}]$, with $Q^{2}=\omega^{2}-q^{2}$ and $\tilde{Q}^{2}=-q^{2}$. In the local frame, where the flow velocity is $u=(1, \mathbf{0}), \omega$ and $\mathbf{q}$ become the time and spatial components of the vector. Similarly, one also defines a tensor orthogonal to $u_{\mu}$,

$$
\tilde{g}_{\mu \nu}=g_{\mu \nu}-u_{\mu} u_{\nu} .
$$

The full gluon propagator with momentum $Q$ is obtained from the vacuum polarization by using Dyson's equation,

$$
\Delta^{\mu \nu}=\frac{\mathcal{P}_{T}^{\mu \nu}}{-Q^{2}+\Pi_{T}}+\frac{\mathcal{P}_{L}^{\mu \nu}}{-Q^{2}+\Pi_{L}}+(\alpha-1) \frac{Q^{\mu} Q^{\nu}}{Q^{4}},
$$

where $\alpha$ is a gauge fixing parameter, the longitudinal $\mathcal{P}_{L}^{\mu \nu}$, and transverse tensor $\mathcal{P}_{T}^{\mu \nu}$ are defined as

$$
\begin{aligned}
\mathcal{P}_{L}^{\mu \nu} & =\frac{-1}{Q^{2} q^{2}}\left(\omega Q^{\mu}-Q^{2} u^{\mu}\right)\left(\omega Q^{\nu}-Q^{2} u^{\nu}\right), \\
\mathcal{P}_{T}^{\mu \nu} & =\tilde{g}^{\mu \nu}+\frac{\tilde{Q}^{\mu} \tilde{Q}^{\nu}}{q^{2}},
\end{aligned}
$$

which are orthogonal to $Q^{\mu}$ and also to each other, i.e.,

$$
Q_{\mu} \mathcal{P}_{L}^{\mu \nu}=Q_{\mu} \mathcal{P}_{L}^{\mu \nu}=\mathcal{P}_{L \nu}^{\mu} \mathcal{P}_{T}^{\nu \rho}=0 .
$$

In additon, one also has $\mathcal{P}^{\mu \rho} \mathcal{P}_{\rho \nu}=\mathcal{P}_{\nu}^{\mu}$. The free gluon propagator at zero temperature is in this case

$$
D^{\mu \nu}=\left(-g^{\mu \nu}+\alpha \frac{Q_{\mu} Q_{\nu}}{Q^{2}}\right) \frac{1}{Q^{2}} .
$$

If we choose Feynman gauge $(\alpha=1)$, the full propagator also satisfies $Q_{\mu} \Delta^{\mu \nu}=0$. The transverse and longitudinal self-energies are 21

$$
\Pi_{L}(Q)=\left(1-x^{2}\right) \pi_{L}(x), \Pi_{T}(Q)=\pi_{T}(x)
$$

with the scaled self-energies, $\pi_{L}(x)$ and $\pi_{T}(x)$, given in Eqs. (9) and (10), where $x=\omega / q$.

Using the full gluon propagator, $\Delta^{\mu \nu}$, we can obtain the effective matrix element of quark scatterings $q_{i}\left(P_{1}\right)+q_{j}\left(P_{2}\right) \rightarrow q_{i}\left(P_{3}\right)+q_{j}\left(P_{4}\right)(i \neq j)$,

$$
\begin{aligned}
\left|\mathcal{M}_{q q}\right|^{2}= & C_{q q} g^{4} 4\left\{4\left|P_{1} \cdot \Delta \cdot P_{2}\right|^{2}-2\left(P_{1} \cdot P_{3}\right)\left(P_{2} \cdot \Delta \cdot \Delta^{*} \cdot P_{4}\right)\right. \\
& \left.-2\left(P_{2} \cdot P_{4}\right)\left(P_{1} \cdot \Delta \cdot \Delta^{*} \cdot P_{3}\right)+\left(P_{1} \cdot P_{3}\right)\left(P_{2} \cdot P_{4}\right)|\Delta|^{2}\right\},
\end{aligned}
$$

where $C_{q q}=\left(N_{c}^{2}-1\right) / 4 N_{c}^{2}=2 / 9$ is the color factor and

$$
\begin{aligned}
\Delta^{\mu \rho} \Delta_{\rho}^{* \nu} & =\frac{\mathcal{P}_{T}^{\mu \nu}}{\left|Q^{2}-\Pi_{T}\right|^{2}}+\frac{\mathcal{P}_{L}^{\mu \nu}}{\left|Q^{2}-\Pi_{L}\right|^{2}} \\
|\Delta|^{2}=\Delta^{\mu \nu} \Delta_{\nu \mu}^{*} & =\frac{2}{\left|Q^{2}-\Pi_{T}\right|^{2}}+\frac{1}{\left|Q^{2}-\Pi_{L}\right|^{2}} .
\end{aligned}
$$


For small angle scatterings, $-Q^{2} / 2=P_{1} \cdot P_{3}=P_{2} \cdot P_{4} \ll P_{1} \cdot P_{2}=P_{3} \cdot P_{4}$, only the first term, corresponding to t-channel scattering, in Eq. (65) is dominant. Furthermore, $\omega=x q \approx \mathbf{v}_{\mathbf{1}} \cdot \mathbf{q} \approx \mathbf{v}_{\mathbf{2}} \cdot \mathbf{q}$ from the energy and momentum conservation. In this approximation, one can verify that

$$
\begin{aligned}
& P_{1} \cdot \mathcal{P}_{L} \cdot P_{2}=-E_{1} E_{2}\left(\mathbf{v}_{1} \cdot \mathbf{v}_{2}-x^{2}\right), \\
& P_{1} \cdot \mathcal{P}_{T} \cdot P_{2}=E_{1} E_{2}\left(1-x^{2}\right) .
\end{aligned}
$$

Define $\cos \phi=\left(\mathbf{v}_{1} \times \hat{\mathbf{q}}\right) \cdot\left(\mathbf{v}_{1} \times \hat{\mathbf{q}}\right)$, we can express $\mathbf{v}_{1} \cdot \mathbf{v}_{2}$ as

$$
\begin{aligned}
\mathbf{v}_{1} \cdot \mathbf{v}_{2} & =\left(\mathbf{v}_{1} \cdot \hat{\mathbf{q}}\right)\left(\mathbf{v}_{2} \cdot \hat{\mathbf{q}}\right)+\cos \phi \sqrt{1-\left(\mathbf{v}_{1} \cdot \hat{\mathbf{q}}\right)^{2}} \sqrt{1-\left(\mathbf{v}_{2} \cdot \hat{\mathbf{q}}\right)^{2}} \\
& =x^{2}+\left(1-x^{2}\right) \cos \phi .
\end{aligned}
$$

We have then

$$
\left|\mathcal{M}_{q q}\right|^{2} \approx C_{q q} g^{4} 16\left(E_{1} E_{2}\right)^{2}\left|\frac{1-x^{2}}{\omega^{2}-q^{2}-\Pi_{L}}-\frac{\left(1-x^{2}\right) \cos \phi}{\omega^{2}-q^{2}-\Pi_{T}}\right|^{2} .
$$

The matrix elements for gluon-quark and gluon-gluon scatterings are similar in the small angle approximation, except the color factors, $C_{g q}=1 / 2$ and $C_{g g}=N_{c}^{2} /\left(N_{c}^{2}-\right.$ $1)=9 / 8$. For scatterings of identical particles, one should also multiply a factor of 2 to take into account of the equal contributions of $t$ and $u$-channel scatterings. Using $E_{3} \approx E_{1}, E_{4} \approx E_{2}$, and Eq. (64) one arrives at Eq. (8).

\section{B Calculation of the relaxation time}

For a system near thermal equilibrium, the energy-momentum tensor is given to the zeroth order of the deviation $\delta f$ by

$$
T_{(0)}^{\mu \nu}=(\epsilon+P) u^{\mu} u^{\nu}-g^{\mu \nu} P,
$$

where $\epsilon$ is the energy density and $P$ the pressure. One can also split the derivative $\partial_{\mu}$ into components parallel and orthogonal to the flow velocity $u^{\mu}$,

$$
\partial_{\mu}=u_{\mu} D+\tilde{\partial}_{\mu}
$$

where $D=u \cdot \partial$ and $\tilde{\partial}_{\mu}=\partial_{\mu}-u_{\mu} D$. The energy-momentum conservation $\partial_{\nu} T_{(0)}^{\nu \mu}=0$ can be rewritten as

$$
\begin{aligned}
D \epsilon+(\epsilon+P) \tilde{\partial} \cdot u & =0, \\
(\epsilon+P) D u^{\mu}-\tilde{\partial}^{\mu} P & =0 .
\end{aligned}
$$

Conservation of entropy requires that the entropy density $s=(\epsilon+P) / T$ fulfills $\partial_{\mu}\left(s u_{\mu}\right)=D s+s \tilde{\partial} \cdot u=0$. Thus we obtain

$$
\frac{D T}{T}=-\frac{\partial P}{\partial \epsilon} \tilde{\partial} \cdot u
$$


where $\partial P / \partial \epsilon=1 / 3$ for an ideal gluon gas.

We assume the space-time variation of the distribution $f$ is small. To the leading order in a gradient expansion in the relaxation time approximation, we have

$$
\delta f=-\frac{\theta(p)}{p \cdot u} p \cdot \partial f^{\mathrm{eq}}=\frac{\theta(p)}{p \cdot u} f^{\mathrm{eq}}\left(1 \pm f^{\mathrm{eq}}\right) p \cdot \partial\left(\frac{p \cdot u}{T}\right) .
$$

Using the hydrodynamical relations, Eqs. (74) and (75), one can rewrite the above as

$$
\frac{\delta f}{f^{\mathrm{eq}}\left(1 \pm f^{\mathrm{eq}}\right)}=\frac{\theta(p)}{T} p^{\mu} X_{\mu}+\frac{\theta(p)}{T} \Sigma^{\mu \nu} U_{\mu \nu},
$$

where $U_{\mu \nu}=(1 / 2)\left(\tilde{\partial}_{\mu} u_{\nu}+\tilde{\partial}_{\nu} u_{\mu}\right), X_{\mu}=\tilde{\partial}_{\mu} P /(\epsilon+P)-\tilde{\partial}_{\mu} T / T$ which vanishes for an ideal quark and gluon gas and $\Sigma_{\mu \nu}$ is another tensor orthogonal to the flow velocity,

$$
\Sigma^{\mu \nu}(p)=(p \cdot u)\left[\frac{\tilde{p}^{\mu} \tilde{p}^{\nu}}{(p \cdot u)^{2}}+\frac{1}{3} \tilde{g}^{\mu \nu}\right] .
$$

To leading order in the gradient expansion, the Boltzmann equation becomes

$$
\begin{aligned}
f_{1}^{\mathrm{eq}}\left(1 \pm f_{1}^{\mathrm{eq}}\right) \Sigma^{\mu \nu}\left(p_{1}\right)= & \nu_{g} \int d p_{2} d p_{3} d p_{4}\left[\theta\left(p_{1}\right) \Sigma_{1}^{\mu \nu}+\theta\left(p_{2}\right) \Sigma_{2}^{\mu \nu}-\theta\left(p_{3}\right) \Sigma_{3}^{\mu \nu}-\theta\left(p_{4}\right) \Sigma_{4}^{\mu \nu}\right](79) \\
& f_{1}^{\mathrm{eq}} f_{2}^{\mathrm{eq}}\left(1 \pm f_{3}^{\mathrm{eq}}\right)\left(1 \pm f_{4}^{\mathrm{eq}}\right) \frac{1}{2}\left|M_{12 \rightarrow 34}\right|^{2}(2 \pi)^{4} \delta^{4}\left(P_{1}+P_{2}-P_{3}-P_{4}\right) .
\end{aligned}
$$

In general, the relaxation time is momentum dependent and it, or the deviation from local equilibrium, must be determined by solving the Boltzman equation. As a first approximation, we assume $\theta$ is independent of the momentum $p$ (one can also assume $\theta \propto p$ which is found to be a good approximation in calculations of viscosities [17]). Multiplying both sides of the above equation by $\Sigma^{\mu \nu}\left(p_{1}\right)$ and integrating over $d p_{1}$, we have then

$$
\theta=\frac{\int d p f^{\mathrm{eq}}\left(1 \pm f^{\mathrm{eq}}\right) \Sigma^{2}(p)}{\mathcal{L}[\Sigma]}
$$

where

$$
\begin{aligned}
\mathcal{L}[\Sigma]= & \frac{\nu_{g}}{(2 \pi)^{8}} \int d^{3} p_{1} f_{1}^{\mathrm{eq}}\left(1 \pm f_{3}^{\mathrm{eq}}\right) \int d^{3} p_{2} f_{2}^{\mathrm{eq}}\left(1 \pm f_{4}^{\mathrm{eq}}\right) \int d^{3} q \int d \omega \frac{1}{4}\left(\Sigma_{1}+\Sigma_{2}-\Sigma_{3}-\Sigma_{4}\right)^{2} \\
& C_{g g} g^{4}\left|\frac{1}{q^{2}+\pi_{L}(x)}-\frac{\left(1-x^{2}\right) \cos \phi}{q^{2}\left(1-x^{2}\right)+\pi_{T}(x)}\right|^{2} \delta\left(\omega-\mathbf{v}_{\mathbf{1}} \cdot \mathbf{q}\right) \delta\left(\omega-\mathbf{v}_{\mathbf{2}} \cdot \mathbf{q}\right)
\end{aligned}
$$

where again we have made the approximation of dominance of small angle scatterings in the local frame, in which $u=(1,0)$ and $\Sigma_{\mu \nu}$ only has nonvanishing spatial components,

$$
\Sigma_{i j}=\frac{1}{E}\left(p_{i} p_{j}-\frac{1}{3} E^{2} \delta_{i j}\right), \text { and } \Sigma^{2}=\frac{2}{3} E^{2} .
$$

Using Eq. (6) we have

$$
\left(\Sigma_{1}+\Sigma_{2}-\Sigma_{3}-\Sigma_{4}\right)_{i j} \approx\left(v_{2}-v_{1}\right)^{i} q^{j}+\left(v_{2}-v_{1}\right)^{j} q^{i}-\omega\left(v_{2}^{i} v_{2}^{j}-v_{1}^{i} v_{1}^{j}\right)
$$


for small $q$ and $\omega$. In terms of $\cos \phi$ and $x=\omega / q$,

$$
\begin{aligned}
\left(\Sigma_{1}+\Sigma_{2}-\Sigma_{3}-\Sigma_{4}\right)^{2} & =2\left(1-\mathbf{v}_{1} \cdot \mathbf{v}_{2}\right)\left[2 q^{2}-2 \omega \mathbf{q} \cdot\left(\mathbf{v}_{1}+\mathbf{v}_{2}\right)+\omega^{2}\left(1+\mathbf{v}_{1} \cdot \mathbf{v}_{2}\right)\right] \\
& =2 q^{2}(1-\cos \phi)\left(1-x^{2}\right)^{2}\left(2-x^{2}+x^{2} \cos \phi\right)
\end{aligned}
$$

One can easily complete the angular integrations in $\mathcal{L}[\Sigma]$ similarly as in the calculation of the gluon damping rate, and has

$$
\begin{aligned}
\mathcal{L}[\Sigma] & =\frac{\nu_{g} C_{g g} g^{4}}{(2 \pi)^{5}} \int d p_{1} p_{1}^{2} f_{1}^{\mathrm{eq}}\left(1 \pm f_{1}^{\mathrm{eq}}\right) \int d p_{2} p_{2}^{2} f_{2}^{\mathrm{eq}}\left(1 \pm f_{2}^{\mathrm{eq}}\right) \mathcal{I}\left(q_{\mathrm{max}}, q_{D}\right) \\
\mathcal{I}\left(q_{\max }, q_{D}\right) & =\int_{0}^{q_{\max }^{2}} d q^{2} \int_{-1}^{1} d x q^{2}\left(1-x^{2}\right)^{2}\left\{\frac{1-\frac{3}{4} x^{2}}{\left|q^{2}+\pi_{L}(x)\right|^{2}}\right. \\
& \left.+\frac{1}{2}\left(1-\frac{7}{8} x^{2}\right) \frac{\left(1-x^{2}\right)^{2}}{\left|q^{2}\left(1-x^{2}\right)+\pi_{T}(x)\right|^{2}}+\operatorname{Re}\left(\frac{\left(1-x^{2}\right)^{2}}{\left[q^{2}+\pi_{L}^{*}(x)\right]\left[q^{2}\left(1-x^{2}\right)+\pi_{T}(x)\right]}{ }^{\dagger}\right) 6\right\}
\end{aligned}
$$

After performing integrations like

$$
\int_{0}^{\infty} d p f^{\mathrm{eq}}\left(1+f^{\mathrm{eq}}\right) p^{n}=n ! \zeta(n) T^{n+1}
$$

for a Bose-Einstein distribution $f^{\mathrm{eq}}$, where $\zeta(n)$ is the Riemann's $\zeta$-function, we obtain for the relaxation time,

$$
\frac{1}{\theta}=\frac{5 N_{c}^{2}}{4 \pi} \frac{g^{4}}{(4 \pi)^{2}} T \mathcal{I}\left(q_{\max }, q_{D}\right)
$$

The integral $\mathcal{I}\left(q_{\max }, q_{D}\right)$ has no dimension and therefore should be only a function of $q_{\max } / q_{D}$. Numerical evaluation of the integral gives

$$
\mathcal{I}\left(q_{\max } / q_{D}\right)=2.3 \ln \left(q_{\text {max }}^{2} / q_{D}^{2}\right)-0.62+2.7 \frac{q_{D}^{2}}{q_{\text {max }}^{2}}+\mathcal{O}\left(q_{D}^{4} / q_{\text {max }}^{4}\right)
$$

for large values of $q_{\max } / q_{D}$. Considering Debye screening from only gluon interactions, we have

$$
\frac{1}{\theta} \approx 0.92 N_{c}^{2} \alpha_{s}^{2} T\left[\ln \left(\frac{1.6}{N_{c} \alpha_{s}}\right)+\mathcal{O}\left(\alpha_{f}\right)\right] .
$$

Similarly as we argued after Eq. (20), contributions to the order $\alpha_{s}^{3}$ must be neglected since they are not complete in our calculation.

\section{References}

[1] J.P. Blaizot and A.H. Mueller, Nucl. Phys. B289, 847 (1987).

[2] K. Kajantie, P. V. Landshoff and J. Lindfors, Phys. Rev. Lett. 59, 2517 (1987); K. J. Eskola, K. Kajantie and J. Lindfors, Nucl. Phys. B323, 37 (1989); K. J. Eskola, K. Kajantie and J. Lindfors, Phys. Lett. B 214, 613 (1989). 
[3] X.-N. Wang and M. Gyulassy, Phys. Rev. D 44, 3501 (1991); ibid. 45, 844 (1992); Phys. Rev. Lett. 68, 1480 (1992); Comp. Phys. Comm. 83, 307 (1994).

[4] K. Geiger and B. Müller, Nucl. Phys. B369, 600 (1992); K. Geiger, Phys. Rev. D 47, 133 (1993).

[5] K. J. Eskola and X.-N. Wang, Phys. Rev. D 48, 1275 (1993).

[6] T. S. Biro, E. van Doorn, B. Müller, M.H. Thoma and X.-N. Wang, Phys. Rev. C 48, 1275 (1993).

[7] X.-N. Wang, Phys. Rev. D 43,104 (1991).

[8] H. Heiselberg and X.-N. Wang, hep-ph-9504244.

[9] E. Braaten and R. D. Pisarski, Phys. Rev. Lett. 64, 1338 (1990); Phys. Rev. D 46, 1829 (1992).

[10] E. Braaten and R. D. Pisarski, Phys. Rev. D 42, R2156 (1990).

[11] C. P. Burgess and A. L. Marini, Phys. Rev. D 45, R17 (1992); A. Rebhan, ibid 48, 482 (1992).

[12] H. Heiselberg and C. J. Pethick, Phys. Rev. D 47, R769 (1993).

[13] R. D. Pisarski, Phys. Rev. D 47, 5589 (1993).

[14] P. Danielewicz and M. Gyulassy, Phys. Rev. D 31, 53 (1985).

[15] A. Hosoya and K. Kajantie, Nucl. Phys. B250, 666 (1985).

[16] G. Baym, H. Monien, C. J. Pethick, and D. G. Ravenhall, Phys. Rev. Lett. 64, 1867 (1990).

[17] H. Heiselberg, Phys. Rev. D 49, 4739 (1994).

[18] M. H. Thoma, Phys. Rev. D 49, 459 (1994).

[19] T. S. Biró, B. Müller and X.-N. Wang, Phys. Lett. 283B, 171 (1992).

[20] S. de Groot, W. van Leeuwen and Ch. van Weert, Relativistic Kinetic Theory (North-Holland, 1980).

[21] H. A. Weldon, Phys. Rev. D 26, 1394 (1982).

[22] H. A. Weldon, Phys. Rev. D 28, 2007 (1983).

[23] T. S. Biró and B. Müller, Nucl. Phys. A561, 477 (1993). 
[24] R. D. Pisarski, Phys. Rev. Lett. 63, 1129 (1989).

[25] G. Baym, Phys. Lett. 138B, 18 (1984).

[26] K. Kajantie and T. Matsui, Phys. Lett. 164B, 373 (1985).

[27] B. Banerjee, R. S. Bhalerao and V. Ravishankar, Phys. Lett. 224B, 16 (1989).

[28] S. Gavin, Nucl. Phys. B351, 561 (1991).

[29] R. D. Field, Application of Perturbative QCD, Frontiers in Physics, Vol. 77 (Addison-Wesley, Reading, MA, 1989).

[30] J. D. Bjorken, Phys. Rev. D 27, 140 (1983).

[31] H1 Collaboration, I. Abt et al., Nucl. Phys. B407, 515 (1993); ZEUS Collaboration, M. Derrick et al., Phys. Lett. 316B, 412 (1993).

[32] K. J. Eskola, K. Kajantie and P. V. Ruuskanen, Phys. Lett. 332B, 191 (1994).

[33] Z. Lin and M. Gyulassy, Phys. Rev. C 51, 2177 (1995).

[34] P. Lévai, B. Müller and X.-N. Wang, Phys. Rev. C 51, 3326 (1995).

[35] E. Shuryak, Phys. Rev. Lett. 68, 3270 (1992).

[36] A. Weldon, Nucl. Phys. A566 581c (1994).

[37] L. Xiong and E. Shuryak, Phys. Rev. C 49, 2207 (1994). 